\title{
Imitation as Mechanism for Mimicry
}

Original Study

\author{
Stephen Jarosek \\ Independent Researcher
}

Received: December 2020; Accepted: March 2021

\begin{abstract}
The neo-Darwinian paradigm is unable to account for the resilient, complex forms that evolve in nature and persist across time. Random mutations do not explain the occurrence of organisms that mimic complex forms in often astonishing detail. In the absence of God as creator, or random mutations as the basis for adaptive traits, there is something else going on. The case that I present in this article is that the only possible mechanism for mimicry in nature is imitation.
\end{abstract}

Keywords: biosemiotic; entropy; imitation; mimicry; mutation; neo-Darwinism; pragmatism.

\section{MIMICRY'S RELEVANCE \\ TO PEIRCEAN SEMIOTICS}

It was Thomas Sebeok who introduced into the field of semiotics the idea of mimicry as a semiotic phenomenon. And it was largely under Sebeok's influence that mimicry has come to be associated with iconicity. From Maran (2017a):

Presumably due to the influence of Thomas A. Sebeok's writings, the connection between mimicry and iconicity has been repeatedly expressed in semiotic literature, and in general semiotic overviews, mimicry is often referred to as an example of iconicity in the natural world.

(Maran 2017a, p. 37)

Because of the relevance of mimicry to iconicity, it is also of direct relevance to semiotic theory. Citing Peirce, Maran (2017b) writes,

An icon is a sign whose "qualities resemble those of that object, and excite analogous sensations in the mind for which it is a likeness" (CP 2.229), and "such a sign whose significance lies in the qualities of its replicas in themselves is an icon, image, analogue, or copy. Its object is whatever that resembles it its interpretant takes it to be the sign of, and is as sign of that object in proportion as it resembles it" (MS [R]
7:14/5) [...] In addition to resemblance, a sign also needs to have a representational character-the sign (in a narrow sense, representamen) needs to stand for its object. Thus, when assessing the connection between mimicry and iconicity, we should ask-what is the sign in mimicry and for what does it stand for? (Maran 2017b, pp. 55-56)

Maran's question of what a sign stands for is ultimately a question of pragmatism. How does an agent define the things that matter? To this end, mimicry provides answers that can be vital to an agent's survival. If an agent has no clue how their world should be interpreted (as would be expected in newborns, for example), their first most easily accessible clues are obtained by observing what the surrounding environment, comprised of animate and inanimate objects, is doing. An agent has to know how to $b e$, and mimicry and imitation provide the most efficient ways of addressing the question of being. Knowing how to be relates directly to Firstness, and mimicry/imitation provides the foundation for deciding what choices (relationships) matter. The relationships that matter are the relationships that persist, and mimicry/imitation thus provides a foundation for all three Peircean categories, Firstness, Secondness, and Thirdness (which I generally interpret in the context of motivation, association, and habituation). 
Mimicry has a long history in the context of Darwinian theory as the archetypal example of natural selection. As Kleisner \& Maran (2019) note, "Mimicry thus came to be viewed in modern times as a convincing instantiation of the creative powers of natural selection."

Contrasting with their introduction of mimicry as an example of Darwinian natural selection, Kleisner \& Maran (2019) then draw our attention to the multi-faceted manifestations of mimicry in nature. They provide examples of the diversity of mimicry, like "molecular mimicry, where a virus imitates the proteins of a host organism, vocal mimicry of birds, and sexual mimicry used for intraspecies communication" to ask what they all share in common. They opt for embracing "the concept of mimicry in its full richness, that is, we prefer thinking of mimicry in sensu lato instead of adopting any of the stricter definitions that have been proposed." They conclude that the theme unifying all the different forms of mimicry is meaning, writing; "Meaning' is the condition sine qua non for the existence of mimicry." And, it is at this point that Kleisner \& Maran's rationale intersects with my own term, assimitation, which I define in Jarosek (2020). We thus bring to bear on the broader implications of mimicry (assimitation), "not only within its biological, behavioural, and informational connotations, but also in its anecdotal, social, historical, cultural, and artistic contexts" (Kleisner, Maran, 2019).

\section{IMITATION AS THE MECHANISM OF MIMICRY}

Within the context of Darwinian natural selection, mimicry is generally assumed to have a defensive purpose. For example, if a mimic that looks like something other than what predators prey on, it won't be eaten, and is likely to survive to create offspring. Thus, a butterfly that looks like a leaf instead of a butterfly will not be eaten by birds whose diet includes butterflies. Leaf-mimicry is therefore an adaptive trait.

But the contemporary interpretation of Darwinism, with its neo-Darwinian emphasis on genetic mutations, introduces assumptions that are problematic, particularly from the perspective of entropy. That a butterfly that can so perfectly mimic a leaf can be attributed to a random mutation is not intuitively self-evident. Where's the evidence for random mutations as the source of this resilient complexity which persists across time? Mathematically, the likelihoods are problematic, given the multiple variables that Byles (1972) lists that need to be met. Experimentally, it has not been proven.

The ability of complexity to persist across time is a critical concern that is all too often not taken seriously enough. Complexity is one thing. Complexity that persists despite the entropy that assails it from every direction is quite another. The famous infinite-monkey theorem suggests that given enough time, an infinite number of monkeys typing at random will deliver a work of Shakespeare. But, this omits the reality that a very precious Shakespearean masterpiece needs to be completed before the death of the star under whose light it takes place. It needs to survive floods and fire, theft, and accident. The paper on which it is typed needs to survive dampness, bugs, and rot. An infinite number of monkeys need to have their ink and paper replenished and their typewriters maintained. And so on. The ability for complexity to persist across time is non-trivial. The reason it is overlooked relates to the constellation of assumptions that ground our established, neo-Darwinian paradigm. With the narrative of mutation-driven natural selection, there is much baggage to leave at the door. As for the infinite-monkey theorem, so too, when we factor in the points raised by Byles (1972), there are multiple variables that need to come together before mutation-based natural selection can be taken seriously. The idea, therefore, that a random mutation can account for a butterfly that looks like a leaf, one that survives ecosystem threats to persist across multiple generations, is inconsistent with the odds.

Alexander (2019) argues that "some cases of so-called mimicry are probably merely look-a-likes and do not gain an advantage due to their similarity in appearance to something else" (Alexander 2019, 39). She makes the case that many examples of mimicry play no role in natural selection, given that they do not always confer a survival advantage, and she uses the term "look-a-likes" to distinguish them from classic text-book mimicry (as understood in the context of natural selection). This is not inconsistent with my own thesis, which is that neo-Darwinian natural selection, with mutations providing the basis by which complex forms and patterns are replicated, is insufficient to explain mimicry.

However, Alexander opts for a saltationist interpretation (as opposed to gradualist), where evolution takes place with the introduction of an abrupt change in a population, within a single generation, and independently of the pressures of natural selection. This interpretation of mimicry is still problematic, however, because it fails to address the entropy problem. The odds of a dead-leaf butterfly coming into existence by chance are still too remote to satisfy the laws of probability. With folded wings looking spectacularly like a dead leaf, down to the finest detail, with leaf stem, veins, and markings, it is difficult to envisage such level of detail occurring randomly and then persisting across time and across generations. Such a notion defies the odds. There is something else going on.

With the purpose of addressing these sorts of shortcomings, in Jarosek (2020) I introduce imitation as a necessary, fundamental principle for the biosemiotic paradigm; and its application is just as relevant in the case of mimicry.

\section{IMITATION AS PRAGMATISM}

In Jarosek (2020) I provide the rationale for regarding imitation as axiomatic to the life sciences. More specifically, this is a nuanced interpretation, within a semiotic context with reference to Peirce's pragmatism, and quite distinct from its usual interpretation in the sense of 
simple, uncontextualized copying/replication. Thus, imitation is one of the ways in which an agent establishes the things that matter to it. To this end, it was necessary to invent a new word for imitation that incorporates this consideration. Assimitate (assimitation) is a synthesis of the words assume and imitate, and it factors in the semiotic context that motivates an agent to imitate. From here, having defined our terms, we now proceed on the assumption that the case for assimitation as axiomatic to the life sciences has been settled.

In Jarosek (2020), I describe the problem with the neoDarwinian interpretation, particularly with its failure to address entropy. Given the limitations of genetic causation, from the bottom up, there has to be an alternative organizing principle to replace it. That principle operates from the top down, and it is assimitation.

Why is assimitation so important? In the absence of a genetic blueprint to explain behaviour, an agent acquires its cues for being from outside of itself. And it is the external cues that inform an agent how to be. Knowing how to be is fundamental to all life, and it is assimitation that provides the most reliable cues that inform an agent what works. This approach includes the following implications:

- Mimicry in nature, like the butterflies whose folded wings resemble dead leaves, or the stick insects that resemble the sticks and twigs of the plants that they inhabit, are further examples of assimitation (Jarosek 2020). Mimics rely on the objects of their environment to provide them with clues for being, the cues for knowing how to be;

- Octopuses are proficient in the art of camouflage, with their ability to change their colouring to match their surroundings (Prager 2000). They can do this because they have chromatophores under the skin that are connected to the nervous system. In conjunction with their acute vision, octopuses are adept at controlling their chromatophores to match not only background colours, but also the light intensity of their surroundings. They can also modify their skin texture to mimic rocks, sand, and other forms, by altering the papillae on their skin. This raises an important question. If octopuses can choose to assimitate surrounding patterns and textures for camouflage, then why can't other creatures likewise assimitate their surroundings in the course of growing to maturity, and becoming familiar with their ecosystems?

- Why would some agents be predisposed to assimitating inanimate objects? Insects, such as butterflies and stick insects, don't have parents to nurture them. In the absence of parents to assimitate, they look to inanimate objects for their cues. Within the Darwinian narrative, mimicry of this kind is widely understood as being adaptive from a defensive perspective; a stick insect, for example, will be camouflaged from the view of predators. What if, however, this defensive outcome is an incidental side-benefit of the primary dynamic, that being knowing how to be? What are the primary motivations for defining what matters (pragmatism)? What are the primary motivations for imitation? Survival is one such motivator, and assimitation of twigs (for stick insects) is a reliable strategy. That is to say, the mere act of knowing how to be a twig protects stick insects from predators that have no interest in twigs;

- Why assimitate leaves on the ground? Because they are a source of food, warmth, comfort, and familiarity ... to the dead-leaf butterfly. Agents seek to assimitate that which they are comfortable with. People do it, in seeking out the company they keep with friends, and they do it when they nostalgically seek out contexts that remind them of the past;

- In a deeper sense, one might say that an agent wants to "be" what it desires or what it respects. The king of Uganda wants to be a lion, and likes to assimitate it:

The king was regarded as a lion or a leopard, whether because the animal was thought to be his ancestor, or simply that he shared its qualities without being directly descended from it. His lion- or leopard-nature meant that he, like these animals, had to kill. It was right and proper for him to kill, to spread terror as these animals did; his propensity for killing was inborn.

(Canetti 1973, 423)

Extending this rationale to insects, a dead-leaf butterfly wants to be the source of its comfort ... a leaf. Emphasis here is on the broader definition of assimitation that subsumes mimicry within its scope. We are also taking our cue from Kleisner \& Maran (2019), who want to identify what all the different forms of mimicry have in common-that is, "not only within its biological, behavioural, and informational connotations, but also in its anecdotal, social, historical, cultural, and artistic contexts." Thus the king of Uganda wanting to be a lion is a suitable metaphor for the butterfly wanting to be a leaf, and provides a relevant expression for an agent's primal motivation to identify with elements of the environment;

- Does it make rational sense for agents to assimitate inanimate objects? It does when there is no bottom-up (genetic) determinant of behaviour. Humans do it. Computer programmers, spending all their time with computers, arguably, take their cues for being from computers. I would even suggest that neo-Darwinism is itself, an extension of this proclivity to assimitate inanimate objectivity, and the mathematics and linear logic that calculate it, to incorporate it into a theory of living entities;

- There is no such thing as instinct. Every agent has "free will," every agent needs to decide between options, and this is why every agent needs to know how to be. What is frequently mischaracterized as "instinct" is more accurately interpreted as simple 
choices made from a reduced horizon of options. The horizon of options of a fish in a pond is very much reduced in comparison to the horizon of options that is available to a human in culture;

- The domestication of animals as pets can be reinterpreted as agents with animal mind-bodies acquiring their knowing how to be within the context of human cultures. No non-human animal, with neither hands nor vocal chords, can ever learn complex human languages, or learn to drive a car, or learn to eat with a fork and knife. They are not physiologically equipped to define things to matter as humans define them. But, they can learn human civility within human culture;

- The domestication of animals has its equivalent dynamic playing out in the feralization of children, which I explore in some detail in Jarosek (2020). Where non-human animals can know how to be within the context of human cultures, feral children obtain their knowing how to be within the context of animals in the wild that raise them;

- $\quad$ At GIBS 2020 (Lacková, L'., Rodríguez H., C. J., Kull, K. (Eds.) 2020), David Ellison's presentation discussed anthropomorphism, why it should not be regarded as a serious impediment in biosemiotics, and why it cannot be avoided when trying to establish how animals interpret their worlds. Consistent with my own thesis regarding assimitation, we might restate the preceding two examples as follows. One has to be raised by wolves in order to understand wolves (refer to McCrone 1993 on the well-documented case of Victor of Aveyron). And, from the opposite perspective, in domestication an animal has to be raised by humans in order to understand humans;

- Biosemiotics and assimitation, within the context of knowing how to be, can be applied to explain much about gender roles in human culture. Peirce's categories (Firstness, Secondness and Thirdness) are still important, but within the context of biosemiotic theory, they are better understood as the filter through which organisms decide what to imitate. Humans with male mind-bodies are predisposed to assimitating men, and humans with female mind-bodies are predisposed to assimitating women (Jarosek 2020). Analogously, butterflies with delicate, leaf-shaped wings, foraging among leaves on forest floors, are predisposed to assimitating dead leaves. And pets, with soft fur and social natures, are predisposed to assimitating their owners;

- Assimitation, with its relationship to knowing how to be, provides an alternative, very different explanation for the inheritance of behaviour, which I outline in Jarosek (2020). Children first inherit their behaviour not through DNA, but through assimitating their parents. And they assimitate, from their cultures, the habits that exemplify the cultural norms and traditions into which they are immersed, beyond family. Thus, the inheritance of behaviour takes place "down on through the millennia, from mother and father to daughter and son, from generation to generation. In this way, culture attains continuity across time, across centuries" (Jarosek 2020, 49). In a similar way, non-human animals assimitate cues beyond themselves, in order to know how to be, and their offspring continue the "traditions" in the ecosystems (and/or simplified cultures) in which they are immersed.

\section{LAMARCKISM AND THE CASE FOR BIOLOGICAL PLASTICITY}

In Lamarckism, or Lamarckian adaptation, an organism acquires characteristics through use or disuse throughout its life and passes on these characteristics to its offspring. The classic example of Lamarckism that is often cited in the literature is the giraffe's long neck. Implied in Lamarckism is some manner of biological plasticity (which these days is referenced in the context of developmental plasticity). That is, the giraffe acquires its longer neck through reaching for the higher leaves in a tree, and the effort habituates the form that gets passed on to subsequent generations.

Developmental plasticity is well documented within the plant and animal kingdom, though up until recent developments (discussed in further detail below), has failed to consolidate into a robust paradigm. From Lea et al (2017):

The capacity of genetically similar individuals to produce substantially different phenotypes depending upon environmental conditions during early life (defined here as the period between conception and reproductive maturation, following Lindström (1999)) is known as 'developmental plasticity.' Because the impact of early conditions can be so dramatic, with potent effects on reproduction and survival, developmental plasticity is of central interest to multiple disciplines.

(Lea et al. 2017, p. 163)

Many examples of developmental plasticity exist, but we take them for granted because they have not been formalized into a robust theory. For example:

- When we exercise at the gym, we rely on plasticity to build our muscles and to maintain fitness;

- The healing of wounds is a form of biological plasticity;

- When astronauts return after spending considerable time in zero gravity, they face serious acclimatization problems on account of their biological plasticity. Reduced muscle mass, reduced bone density, changes to chromosomes, etc, are all different manifestations of biological plasticity;

- Lea et al. (2017) cite the impact that in utero and early life experiences, for example relating to nutrition, famine, or trauma, can have on obesity, heart disease, schizophrenia, and longevity. 
The topic of developmental plasticity fits within the narrative of systems theory, and the idea of the body as being comprised of colonies of cells that begin as stem cells and differentiate into their respective roles with an organism's growth and development. The assumption in established discourse has been, however, that differentiation is a one-way process, and that that once a body's cells have differentiated into their respective roles within the body, the process cannot be reversed. That situation has now changed in the face of recent research.

Contrary to long-held assumptions, recent studies show that cells are remarkably plastic. Skipper et al (2010) introduce articles for Nature that explore the topic from a number of perspectives, noting that "the molecular and cellular mechanisms that underlie this plasticity is a dynamic area of biology and one that holds great promise for developing new therapies" (Skipper et al, 2010, 703). Kraft \& Rubin (2016) further explore the implications of cellular plasticity, in light of these recent developments. Both papers revolve around the work of Nobel laureates John Gurdon and Shinya Yamanaka, whose work constitute a paradigm shift on the topic of cellular plasticity. Also referenced in both papers is the seminal research of Helen Blau, who introduced the term plasticity, and provided the basis for reconceptualizing the genetics of cellular differentiation with reference to the work of Gurdon and Yamanaka. Kraft \& Rubin (2010) summarize the essence of this insight into cellular plasticity:

Blau went on in 1991 to argue that the fully differentiated cell represented an actively maintained state. That is to say, cell identity was continuously maintained or held in place by regulatory factors (Blau and Baltimore, 1991). This was a central plank in a novel conception of the cell, premised on the idea that cell identity was not fixed but, rather, was something that could change in response to specific signals. Here, the specialised cell did not represent a biological endpoint, but rather represented one of many possible cell identities-possibilities realised through the process of cell differentiation.

(Kraft, Rubin 2010, p. 499)

While the aforementioned research has focused principally on plasticity in the context of cellular differentiation, with cells redefining their roles within the body, it has broader implications in the context of "swarming," and the idea that a body is a collective that in effect, can be said to "swarm" towards the options that are available to it, in its ecosystem, or Umwelt. In the context of Lamarckism, it is this latter interpretation in which we are interested, and neural plasticity is an important category of cellular plasticity. If we can demonstrate "swarming" in the context of neural plasticity, then it is not unreasonable to extend the swarming metaphor more generally, to other cell types.

In Jarosek (2013 and 2020) I make a clear distinction between strong neural plasticity and weak neural plasticity. Strong neural plasticity, with associative learning and habituation playing a fundamental role in neural behavior, conforms to Sharov's implementation of agency theory (Sharov, 2018). Weak neural plasticity, by contrast, does not. Strong neural plasticity provides a more realistic account of the role of experience in establishing the functional specializations in the brain. This contrasts with weak neural plasticity, which regards the brain's functional specializations as being largely determined in the genetic code, and is an extension of the neo-Darwinian narrative, with its emphasis on instinct.

With reference to a case documented in Lancet (Feuillet et al. 2007), describing a man with severe hydrocephalus (water on the brain) who was able to function normally, despite massive ventricular enlargement resulting in a 50 to $75 \%$ reduction in brain volume, I concluded that this could not have been possible without strong neural plasticity. That is, the brain as a colony of neurons can be thought of as "swarming" around its options:

This suggests that functional specializations in the brain are not confined to a developmental trajectory spelled out in a genetic blueprint but rather, that they self-organize in exactly the same way that any colony of social organisms self-organize in response to pressures from the ecosystem. If water on the brain makes regions of the brain uninhabitable, then neurons will set themselves up in regions that are habitable.

(Jarosek 2013, p. 215)

Given that assimitation impacts on the habits that can, in turn, impact on cellular (and neural) plasticity, physiology, evolution and Lamarckian adaptation, we conclude that the saltationist interpretation for mimicry is not justified. A gradualist interpretation is more likely, and the acquisition of traits, in conjuction with assimitation, provides a much more likely account of evolution that properly addresses the problem of entropy.

Lamarckism, cellular plasticity and assimitation are integral to an interpretation of Darwinian evolution that properly addresses entropy. Neo-Darwinism, with its bottom-up emphasis on natural selection based on genetic mutations, falls well short.

\section{CONCLUSION}

Neo-Darwinism makes assumptions about mimicry that do not properly factor in entropy. Alexander (2019) suggests an alternative, saltationist mechanism for mimicry that is independent of natural selection. I conclude that between these two possibilities, a third alternative, assimitation in conjunction with cellular plasticity, is the most likely mechanism, because:

- $\quad$ Assimitation properly factors in entropy;

- In the absence of the bottom-up causation assumed in neo-Darwinian determinism, assimitation provides the top-down instructions for an agent learning to survive, and yearning to know how to be; 
- Developmental (cellular) plasticity provides for the phenotypes that adapt to environmental changes in the spirit of gradualism instead of saltationism;

- Assimitation is consistent with Darwinian evolution incorporating Lamarckian adaptation;

In Jarosek (2020), I had shown, by reversal of reasoning, that autism, as imitation deficit, provides evidence of imitation (assimitation) as a fundamental principle. So too, in this article, I suggest an analogous reversal of reasoning. That is, the astonishing fidelity of some forms of mimicry, as per the example of the dead-leaf butterfly (Alexander 2019), defy any possibility of random occurrence. Without invoking God as creator, or random mutations to account for adaptive traits, there can only be one explanation. Examples of mimicry in nature provide their own evidence in support of assimitation as a fundamental principle for the life sciences.

\section{REFERENCES}

Alexander, V. N., 2019. The Mechanism for Mimicry: Instant Biosemiotic Selection or Gradual Darwinian Fine-Tuning Selection? Biosemiotics 12, 39-55. Available at: <https://doi.org/10.1007/s12304-019-09349-9>.

Byles, R. H., 1972 (March). Limiting Conditions for the Operation of the Probable Mutation Effect. Social Biology, 19(1), 29-34. Retrieved March 28, 2021, from: <http://www.tandfonline.com/doi/abs/10.1080/194 85565.1972.9987962>.

Canetti, E., 1973. Crowds and power. London: Victor Gallancz.

Feuillet, L., Dufour, H., Pelletier, J., 2007. Brain of a white-collar worker. The Lancet, 370(9583), 262.

Jarosek, S., 2013. Pragmatism, neural plasticity and mind-body unity. Biosemiotics, 6(2), 205-230.

Jarosek, S. L., 2020. Knowing how to be: Imitation, the neglected axiom. Cybernetics and Human Knowing 27(3), pp. 33-63, available at:

< https://www.ingentaconnect.com/contentone/imp/ chk/2020/00000027/00000003/art00003 >
Kleisner, K., Maran, T., 2019. Introduction to Signs and Communication in Mimicry. Biosemiotics 12, 1-6. Retrieved March 27, 2021, from: <https://link.springer. com/article/10.1007/s12304-019-09352-0>,

<https://doi.org/10.1007/s12304-019-09352-0>.

Kraft, A., Rubin, B. P., 2016. Changing cells: An analysis of the concept of plasticity in the context of cellular differentiation. BioSocieties 11, 497-525. Available at: <https://doi.org/10.1057/s41292-016-0027-y>.

Lacková, L'., Rodríguez H., C. J., Kull, K. (Eds.), 2020. Gatherings in Biosemiotics XX. Tartu Semiotics Library 20., University of Tartu Press.

Lea, A. J., Tung, J., Archie, E. A., Alberts, S. C., 2017. Developmental plasticity: Bridging research in evolution and human health. Evolution, Medicine, and Public Health, 2017(1), 162-175. Available at: <https:// doi.org/10.1093/emph/eox019>.

McCrone, J., 1993. The Myth of Irrationality - The science of the mind from Plato to Star Trek. Macmillan London.

Maran, T., 2017a. Semiotics of Mimicry. In: Mimicry and Meaning: Structure and Semiotics of Biological Mimicry. Biosemiotics, 16, 35-53, Springer, Cham. Available at: <https://doi.org/10.1007/978-3-319-50317-2_4>.

Maran, T., 2017b. Iconicity and Mimicry. In: Mimicry and Meaning: Structure and Semiotics of Biological Mimicry. Biosemiotics, 16, 55-63, Springer, Cham. Available at: <https://doi.org/10.1007/978-3-319-50317-2_5>.

Prager, E. J., 2000. How do squid and octopuses change color? Scientific American, 2000, September 18. Retrieved March 24, 2019, from: <https://www.scientifica merican.com/article/how-do-squid-and-octopuse/>.

Sharov, A., 2018. Mind, Agency and Biosemiotics. Journal of Cognitive Science 19(2), 195-228.

Skipper, M., Weiss, U., Gray, N., 2010. Plasticity. Nature, 465(7299), 703. Retrieved March 28, 2021 from: <https://www.nature.com/articles/465703a.pdf>.

Return to front page $\uparrow$ 